\title{
Clinical Evaluation of UR-System-PARKO for Recovery of Motor Function of Severe Plegic Hand after Stroke
}

\author{
Hirofumi Tanabe ${ }^{1}$, Yoshifumi Morita ${ }^{2}$ \\ ${ }^{1}$ Department of Health Sciences, Shonan University of Medical Sciences, \\ 16-48 Kamishinano, Totsuka-ku, \\ Yokohama, Kanagawa 244-0806, Japan \\ E-mail: hirofumi.tanabe@sums.ac.jp \\ ${ }^{2}$ Department of Electrical and Mechanical Engineering, Nagoya Institute of Technology, \\ Gokiso-cho, Showa-ku, \\ Nagoya, Aichi 466-8555, Japan \\ E-mail: morita@nitech.ac.jp
}

\begin{abstract}
The first author has developed TANABE therapy for severe hemiplegic stroke patients. In this paper we developed a training system (UR-System-PARKO) on the basis of TANABE therapy. The clinical test of the therapeutic effect of the UR-System-PARKO was performed in severe plegic hand. The results show the effectiveness of the training by the UR-System-PARKO for recovery of motor function for finger extension of the severe plegic hand.

Keywords: Training system, Motor function, Finger extension, Spasticity, Hemiplegic stroke patient
\end{abstract}

\section{Introduction}

With Japan's gradual advancement towards a so-called superannuated society, the number of people physically challenged by disease and by aging is increasing. The corresponding increase in the workload of therapists has become a social issue.

Moreover, according to proposals for health care reform in the rehabilitation field, a system with empiric treatment for evidence-based medicine (EBM) has to be implemented. This change will be accelerated by introducing technology, such as robotics, mechatronics, information processing, and motion control, into the rehabilitation exercises administered by therapists in order to reduce their workload and to improve the therapeutic effects.

Recently, it has been shown that the motor function of stroke patients may be restored even six months or more after the occurrence of the stroke. ${ }^{1,2}$ However, the severity of hemiplegia after the stroke was moderate in all patients who recovered motor function in these reports. More than half of the stroke survivors have a severe plegic hand with difficulty in extending the fingers. ${ }^{3}$ Therefore, the need for developing a method to treat severe plegic hands is significant.

The first author in an occupational therapist who developed the TANABE therapy for severe hemiplegic stroke patients. With this therapy, he performed repeated facilitation training using his hands on patients to help them recover their motor function, and a good treatment outcome was achieved. ${ }^{4}$ In addition to a book on this technique, he has trained many therapists ${ }^{5}$ (Fig. 1). As a result, many therapists have successfully restored finger movement in patients with severe plegic hands. This facilitation method increases the output of the extensor digitorum muscle force through extension 
exercises of the elbow joint while applying resistance to the tips of the fully extended hemiplegic fingers. In this type of training, the therapist has to spend a lot of time with each patient. Therefore, we developed a rehabilitation support system (UR-System-PARKO) designed to reduce the burden on the therapist for resistance training.

The simplified training system previously developed by us for resistance training of hemiplegic upper limbs was applied to a new support system for the hands. This simplified training system provides unidirectional resistance training. We have also developed a brace for securing the plegic hand to the apparatus. The objective of this study was to verify the effect of training on increasing the output of the extensor digitorum muscle force using the UR-System-PARKO on a severe plegic hand.

\section{Training System for Recovery of Motor Function}

\subsection{The previous training system}

We developed rehabilitation support systems for resistance training of hemiplegic upper limbs, namely UR-System $1^{6,7}$ and UR-System $2^{8}$. The UR-System 2 is referred to as the previous training system in this paper. The previous training system is a force display system with one degree-of-freedom that has a mechanical system and a controller. The mechanical system consists of a brace, a training arm, and a powder brake (SINFONIA TECHNOLOGY CO., Ltd., PRB-2.5H). The patient moves the training arm by himself/herself. The brace is attached to the tip of the training arm, which is used to secure the patient's forearm to the apparatus. The powder brake generates a brake force that serves as the resistance force during training. Because this system is not equipped with motors, it is extremely safe and low cost.

In the earlier study ${ }^{9}$, it was found that the therapist selectively uses four different resistance patterns during resistance training. These patterns were detected from the movement analysis performed during the manual training by the therapist using his hands. The four different resistance patterns, namely a step mode, a slope mode, a wall mode, and a constant mode, are installed in the controller. Control programs based on these results are installed in the controller.

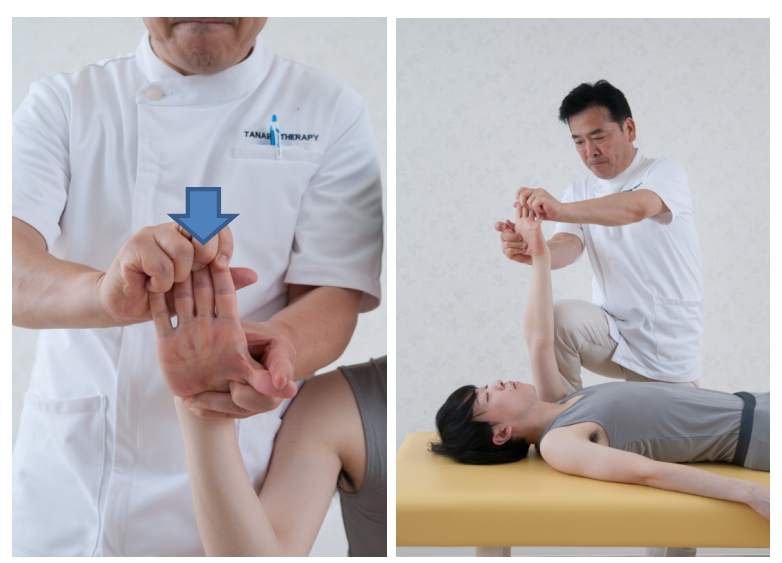

Fig. 1. The author has developed the TANABE therapy for severe hemiplegic stroke patients.

\subsection{Modification of the training system to facilitate finger extension}

In order to improve motor function, especially to facilitate finger extension, an additional device was installed in the training apparatus. A thermoplastic splint for fixation was prepared to fix the plegic hand of a hemiplegic patient in complete extension. Rails were laid on the arm-fixing plate to allow sliding the splint on the plate. Rods were set individually to the index, middle, and ring fingers of the plegic hand, and the hand was set on the arm-fixing plate so that the patient could push the arm with her/her fingertips (Fig. 2).

\section{Clinical Evaluation of Therapeutic Effect}

We conducted clinical tests on patients with severe chronic plegic hands to verify the therapeutic effects of the training with the UR-System-PARKO.

\subsection{Assessment}

The therapeutic effects of the training with the URSystem-PARKO were assessed by using the surface electromyogram (EMG), the modified ashworth scale (MAS), and the active range of motion (A-ROM) test. An EMG electrode pad was applied to the extensor digitorum muscle, and firing of the extensor digitorum muscle during exercise was measured. The MAS is one of the quantitative evaluation methods for spastic paralysis, which is rated in 6 levels. Level 6 indicates no increase in muscle tone.

Level 0 indicates that the affected parts are rigid in either flexion or extension. The A-ROM test is 


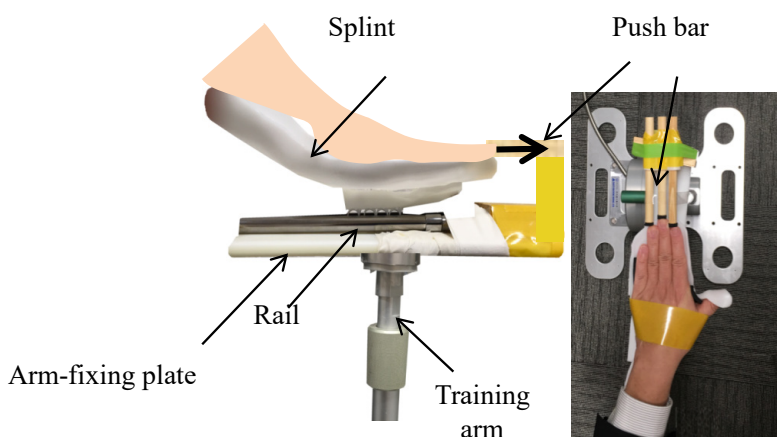

(a) Lateral view (b) View from directly above

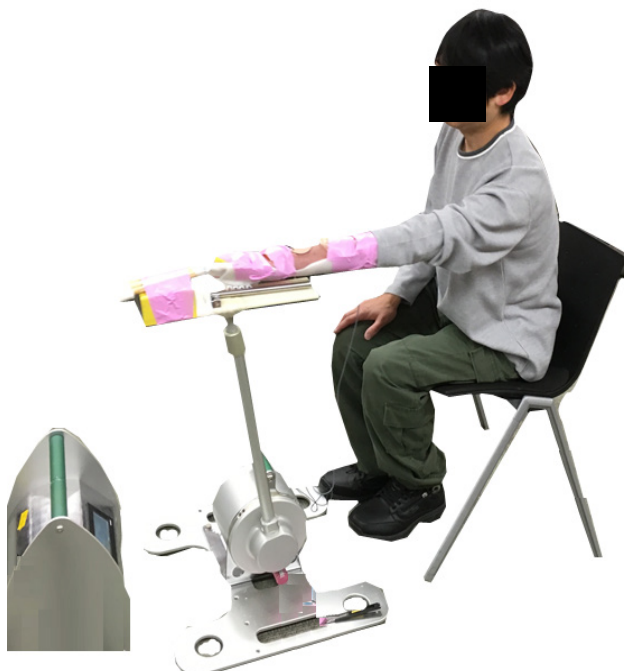

(c) Training

Fig. 2. UR-System-PARKO.

performed to evaluate voluntary motor performance and has been widely used in clinical settings. The A-ROM is measured when a patient moves the joint without any assistance to the muscles surrounding the joint.

\subsection{Patient}

The subject was a hemiplegic patient in his 40 s who had suffered a stroke 8 years ago. The MAS score presenting the grade of spasticity was 3 . (He had a more marked increase in muscle tone through most of the ROM, but the affected part (s) easily moved). The patient did not experience any pain in the training with UR-System-PARKO. The study content was explained and written consent was obtained from the patient.

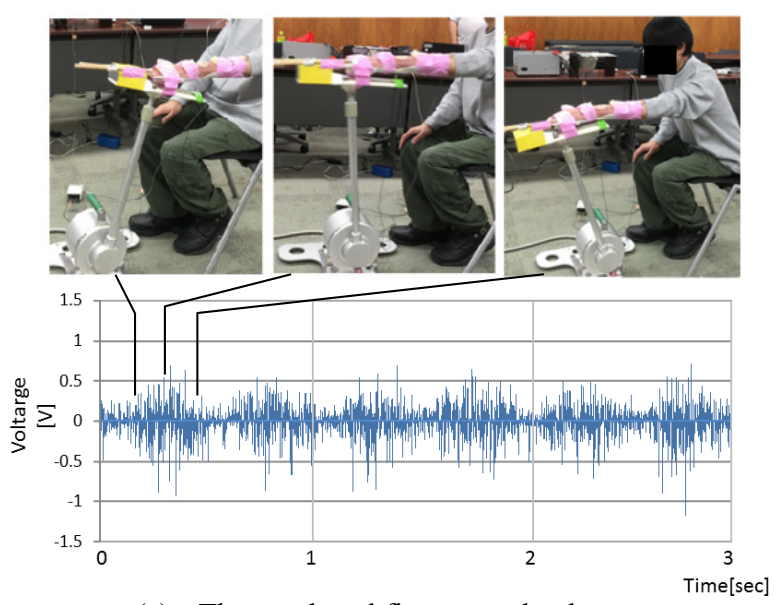

(a) The paralyzed fingers push a lever.

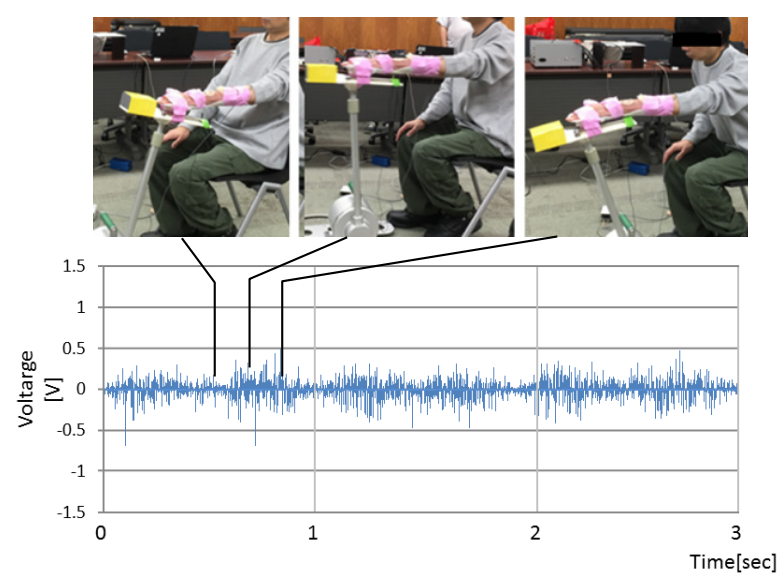

(b) The paralyzed forearm pushes a lever.

Fig. 3. Electromyograms of the extensor digitorum muscle while pushing the lever of the UR-System-PARKO. (Firing increased when the subject pushed the lever with the fingertips of the plegic hand compared with that caused by pushing with the forearm.)

\subsection{Method}

\subsubsection{Setting}

The fingers of the patient were set on the splint in the fully extended position followed by fixation to the arm of the UR-System-PARKO. First, the therapist tried the technique facilitating the extensor digitorum muscle of the plegic hand to confirm the exercise initiation and completion positions and the grade and timing of resistance loading.

Then, the subject's forearm was set on the splint and fixed to the arm-fixing plate of the UR-SystemPARKO. Then, the rods were set to the fingertips of the 
plegic hand to push the lever. The exercise initiation and completion positions, resistance value, and initiation and completion positions of resistance loading confirmed by the therapist beforehand were entered into the UR-System-PARKO using the operating the panel. The resistance value was set at $15 \mathrm{~N}$.

\subsubsection{Comparison of the output of the extensor digitorum muscle force between the positions of applying resistance load}

For manual facilitation treatment of the extensor digitorum muscle, the output of the extensor digitorum muscle force can be increased by loading resistance on the fingertips. The position of pushing the UR-SystemPARKO lever was set at 2 sites: 1 ) the fingertips of the plegic hand and 2) the forearm. The subject pushed the UR-System-PARKO lever against the resistance 10 times with each site, and the muscle force output measured using the EMG was compared between the 2 sites.

\subsubsection{Comparison of the active range of motion and flexor spasticity of the plegic hand between before and after training with the UR-System-PARKO}

Training was set so as to load resistance on the fingertips. The subject moved the hand from the initiation to the completion position and repeated the motion 30 times. A-ROM and MAS were evaluated before initiation and after completion of the exercise training.

\subsubsection{Results and considerations}

On comparison of the output of the extensor digitorum muscle force between the different resistance loading positions, the muscle force output by pushing the lever with the fingertips of the plegic hand was greater than that by pushing with the forearm on visual observation (Fig. 3). The training with the UR-System-PARKO increased the A-ROM of the finger extension when compared with that before training (Fig. 4). The MAS of the finger extensors was 2 before training, and it increased to 4 after training.

\section{Conclusion}

A therapeutic effect of the training with the UR-SystemPARKO was shown. The UR-System-PARKO can improve the motor function of patients with a severe

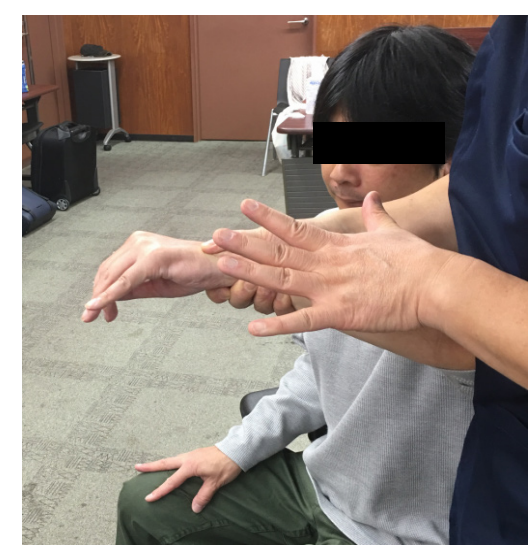

(a) Before training

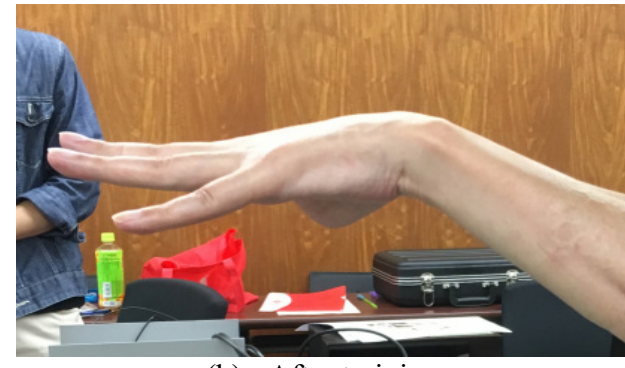

(b) After training

Fig. 4. Finger extension before and after training.

plegic hand after a stroke. The spasticity of the finger extension is reduced after the training. The A-ROMs of the finger extension are expanded after the training.

Our future work is to develop an effective training method using the UR-System-PARKO while considering factors such as number of repetitions, training days, and so on.

This work was supported by JSPS KAKENHI Grant Number JP16K01544.

\section{References}

1. S. L. Wolf, C. J. Winstein, J. P. Miller, P. A. Thompson, E. Taub, G. Uswatte, D. Morris, S. Blanton, D. NicholsLarsen, P. C. Clark, The EXCITE Trial: Retention of Improved Upper Extremity Function Among Stroke Survivors Receiving CI Movement Therapy, Lancet Neurol. 7(1) (2008) 33-40.

2. J. Whitall, S. M. Waller, K. H. Silver, R. F. Macko, Repetitive bilateral arm training with rhythmic auditory cueing improves motor function in chronic hemiparetic stroke, Stroke 31(10) (2007) 2390-2395.

3. H. Nakayama, H. S. Jørgensen, H. O. Raaschou, T. S. Olsen, Recovery of upper extremity function in stroke patients: the Copenhagen Stroke Study, Arch. Phys. Med. Rehabil. 75(4) (1994) 394-398. 
4. H. Tanabe, T. Nagao, R. Tanemura, Application of Constraint-induced Movement Therapy for People with Severe Chronic Plegic Hand, Asian J. Occupational Therapy, 9(1) (2011) 7-14.

5. H. Tanabe, The approach that recovers for a central nerve disorder -TANABE Therapy- (Human Press, Tokyo, 2016).

6. Y. Morita, N. Sato, H. Ukai, H. Tanabe, T. Nagao, R. Tanemura, Y. Takagi and Y. Aoki, Clinical Evaluation of Training System for Recovery of Motor Function after troke in Patients with Hemiplegia, in Proc. 2nd Int. Conf. on NeuroRehabilitation (ICNR2014 in Aalborg, Denmark) (2014), pp.83-92.

7. H. Tanabe, M. Mitsukane, N. Toya, R. Takeichi, H. Hattori, Y. Morita, Y. Takagi, N. Hasegawa, Clinical
Evaluation of UR-System 2 for Recovery of Motor Function of Plegic Upper Limb after Stroke, J. Robotics, Networking and Artificial Life, 3(1), (2016) 33-36.

8. Y. Morita, N. Toya, R. Takeichi, H. Hattori, H. Tanabe, Y. Takagi, N. Hasegawa, Development of 'UR-System 2': a training system for recovery of motor function of plegic upper limb after stroke, Advanced Robotics, 30(21) (2016)

9. Y. Iida, Y. Hiramatsu, K. Yamazaki, Y. Morita, H. Ukai, H. Tanabe, R. Tanemura, K. Yokoyama, Development of resistance training and evaluation support system for upper limbs - Study on resistance training using robot in CI therapy, in Proc. 11th SICE System Integration Division Annual Conf., (2010), pp.1808-1811. (in Japanese). 